Ophthalmologe 2015 $\cdot 112: 942$

DOI 10.1007/s00347-015-0144-1

Online publiziert: 6. Oktober 2015

(c) Springer-Verlag Berlin Heidelberg 2015

CrossMark

L. Daas ${ }^{1} \cdot$ N. Szentmáry ${ }^{1}$ T. Eppig ${ }^{2} \cdot$ A. Langenbucher ${ }^{2} \cdot$ A. Hasenfus ${ }^{3} \cdot$ M. Roth ${ }^{4}$. M. Saeger ${ }^{5} \cdot$ B. Nölle ${ }^{5} \cdot$ B. Lippmann 6 . D. Böhringer ${ }^{6} \cdot$ T. Reinhard $^{6} \cdot$ C. Kelbsch $^{7}$.

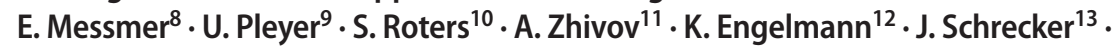
L. Zumhagen ${ }^{14} \cdot$ H. Thieme ${ }^{15} \cdot$ R. Darawsha ${ }^{16} \cdot$ T. Meyer-ter-Vehn $^{17} \cdot$ B. Dick ${ }^{18}$. I. Görsch ${ }^{19} \cdot$ M. Hermel $^{20} \cdot$ M. Kohlhaas ${ }^{21} \cdot$ B. Seitz ${ }^{1}$

${ }^{1}$ Klinik für Augenheilkunde, Universitätsklinikum des Saarlandes UKS, Homburg/Saar, Deutschland

2 Experimentelle Ophthalmologie, Universität des Saarlandes, Homburg/Saar, Deutschland

${ }^{3}$ Institut für Allgemeine und spezielle Pathologie, Universität des Saarlandes, Homburg/Saar, Deutschland

${ }^{4}$ Universitätsaugenklinik, Düsseldorf, Deutschland

${ }^{5}$ Klinik für Ophthalmologie, Universitätsklinikum Schleswig-Holstein, Campus Kiel, Kiel, Deutschland

${ }^{6}$ Klinik für Augenheilkunde, Universitätsklinikum Freiburg, Freiburg, Deutschland

${ }^{7}$ Universitätsaugenklinik, Tübingen, Deutschland

${ }^{8}$ Augenklinik der LMU, München, Deutschland

${ }^{9}$ Universitätsaugenklinik, Berlin Charité, Berlin, Deutschland

${ }^{10}$ Universitätsaugenklinik, Köln, Deutschland

${ }^{11}$ Universitätsaugenklinik, Rostock, Deutschland

${ }^{12}$ Augenklinik Chemnitz, Chemnitz, Deutschland

${ }^{13}$ KKH Rudolf Virchow, Glauchau, Deutschland

14 Universitätsaugenklinik, Münster, Deutschland

${ }^{15}$ Universitätsaugenklinik, Magdeburg, Deutschland

${ }^{16}$ Universitätsaugenklinik, Essen, Deutschland

${ }^{17}$ Universitätsaugenklinik, Würzburg, Deutschland

${ }^{18}$ Universitätsaugenklinik, Bochum, Deutschland

${ }^{19}$ Universitätsaugenklinik, Hamburg-Eppendorf, Deutschland

20 Universitätsaugenklinik, Aachen, Deutschland

${ }^{21}$ Augenklinik Dortmund, Dortmund, Deutschland

\title{
Erratum zu: Das Deutsche Akanthamöbenkeratitis-Register
}

\section{Erste Ergebnisse einer multizentrischen Erhebung}

\section{Erratum zu}

Ophthalmologe 2015 • 112:752-763

DOI 10.1007/s00347-014-3225-7

In diesem Beitrag wurde im Abschnitt Chirurgische Therapie eine Prozentzahl falsch angegeben.

Wir bitten, die korrekte Zahl zu beachten und den Fehler zu entschuldigen.

\section{Chirurgische Therapie}

Wie schwerwiegend diese Keratitisform ist, zeigt sich auch daran, dass in $\mathbf{4 0 , 4} \%$ der Fälle eine PKP durchgeführt wurde.

\section{Korrespondenzadresse}

\section{Daas}

Klinik für Augenheilkunde Universitätsklinikum des Saarlandes UKS Kirrbergerstr. 100, 66424 Homburg/Saar loay.daas@uks.eu 